\title{
Avaliação de carcaça bovina: uma revisão sobre o uso do ultrassom
}

\section{[Bovine carcass evaluation: a review about the use of the ultrasound]}

\section{"Revisão / Review"}

\author{
Marcelo José Ferreira Batista da Silva ${ }^{1 *}$, Leandro Fragoso Lins ${ }^{1}$, Ney Bráulio de Oliveira Lins ${ }^{1}$, Mariana \\ Gomes Ferreira Machado de Siqueira ${ }^{2}$, Andrea Paiva Botelho Lapenda de Moura², \\ Pedro Marinho de Carvalho Neto ${ }^{3}$, Severino Benone Paes Barbosa ${ }^{1}$, Wilson Moreira Dutra Júnior ${ }^{1}$
}

\author{
${ }^{1}$ Departamento de Zootecnia, Universidade Federal Rural de Pernambuco, Recife-PE, Brasil. \\ ${ }^{2}$ Departamento de Medicina Veterinária, Universidade Federal Rural de Pernambuco, Recife-PE, Brasil. \\ ${ }^{3}$ Departamento de Tecnologia Rural, Universidade Federal Rural de Pernambuco, Recife-PE, Brasil. \\ *Autor para correspondência/Corresponding author: E-mail: marcelofbatista@ hotmail.com
}

\begin{abstract}
Resumo
Diversos métodos de avaliação de carcaça bovina vêm sendo utilizados para expressar características relacionadas à carcaça, à qualidade da carne e ao produto final de um determinado sistema de produção. Atualmente, o uso do ultrassom na técnica de ultrassonografia é um desses métodos. Assim, o objetivo desta revisão é informar sobre o uso do ultrassom na avaliação de carcaça bovina, pontuar os principais indicadores de medidas na carcaça e sua avaliação, além de apresentar as vantagens e limitações do seu uso. A ultrassonografia vem sendo usada para quatro finalidades dentro das técnicas de avaliação de carcaças: avaliação da composição corporal quanto à deposição de músculo e gordura; avaliação dos reprodutores e sua progênie; seleção em rebanhos; e rendimento de carcaça para o mercado consumidor. A técnica permite a coleta de três principais medidas de carcaças in vivo: espessura de gordura subcutânea, área de olho de lombo e gordura de marmoreio, onde esses indicadores, feitos por ultrassom, apresentam boas correlações com as medidas de carcaça post mortem. Como benefícios da utilização da técnica tem-se a obtenção em tempo real, de forma rápida e barata, das características de composição de carcaça em animais ainda vivos. As principais limitações são tecnológicas, experiência do operador, e características particular do animal. Portanto, conclui-se que a ultrassonografia é uma ferramenta que tem sido largamente utilizada, sendo sua aplicação de extrema importância na avaliação da condição corporal e na seleção genética, possibilitando assim a produção de um animal que atenda o mercado consumidor.
\end{abstract}

Palavras-Chave: carne bovina; conformação de carcaça; qualidade; ultrassonografia.

\begin{abstract}
Several methods for bovine carcass evaluation have been used to express characteristics related to the carcass, to the meat quality, and to the final product of a specific production system. Currently, the use of ultrasonography is one of these methods. Thus, the aim of this review is to report the use of ultrasound in the evaluation of bovine carcass, to signal the main indicators measured in the carcass and its evaluation, and to present the advantages and limitations of its use. Ultrasonography has been used for four purposes in the evaluation of carcasses: assessment of body composition regarding muscle and fat deposition; assessment of breeders and their progeny; selection in herds; and carcass yield for the consumer market. The technique allows the collection of three main measurements for in vivo carcasses: subcutaneous fat thickness, loin eye area, and marbling fat, where these indicators, obtained via ultrasound, show good correlations with post mortem carcass measurements. As the benefit of the technique used, there is the obtainment, in real time, quickly and cheaply, of carcass composition characteristics in still living animals. The main limitations are technology-related, operator experience, and particular characteristics of the animal. Therefore, we conclude that ultrasonography is a tool which has been widely used, and its application is extremely important in the evaluation of body condition and genetic selection, thus enabling the production of an animal that satisfies the consumer market.
\end{abstract}

Keywords: bovine meat; carcass conformation; quality; ultrasonography. 


\section{Introdução}

No Brasil, o principal reflexo sobre as características da carcaça é a grande heterogeneidade dos sistemas de produção na bovinocultura de corte. Sendo assim, características relacionadas aos aspectos qualitativos e quantitativos da carcaça podem sofrer alterações pela genética, manejo e sistema de terminação.

Outro fator a ser considerado quando se pensa em produção de carne é o mercado consumidor, para quem vender essa carne, que tipo de carne produzir, isso porque, este mercado vem tornando-se muito competitivo e exigente. Além disso, para o sucesso na produção é importante que se tenha quantidade, regularidade e principalmente viabilidade econômica responsável pelo peso de abate do animal.

Os métodos de classificação e tipificação de carcaça bovina vêm sendo utilizados para avaliar a qualidade do produto final de um sistema de produção. A classificação consiste em agrupar em classes, ou grupos, carcaças com características semelhantes, sexo e maturidade. Já a tipificação consiste em diferenciar as classes em tipos ordenados hierarquicamente de acordo com critérios específicos, como maturidade, peso da carcaça, conformação, rendimento, gordura de cobertura, entre outras características de carcaça que permitem tipificá-la de acordo com sua qualidade. Assim, os sistemas de classificação e tipificação são usados para descrever o valor da carcaça em termos de carne magra, rendimento e grau de qualidade útil para a indústria ou o mercado varejista, premiando a qualidade da carcaça.

Diante dessa problemática, vários métodos com o objetivo de avaliar características relacionadas à carcaça e a qualidade da carne foram criados. Para que houvesse a avaliação indireta das características ligadas à carcaça, criou-se uma escala de medidas observadas visualmente, por meio de pontuações (escores), utilizado para estimar o grau de acabamento da carcaça (Dibiasi et al., 2010). Ainda segundo esses mesmos autores os escores visuais de conformação e musculatura podem ser importantes para selecionar animais com maior produção de carne.

Outra opção seria a obtenção post mortem de medidas relacionadas à qualidade e rendimento de carcaça. Embora a obtenção dessas características em animais abatidos seja uma alternativa razoável, ela apresenta dois grandes problemas. O primeiro é que o método requer um teste de progênie no qual os animais devem ser abatidos. $\mathrm{O}$ teste de progênie é um eficiente método de se predizer o mérito genético, mas é dispendioso e demanda longos períodos de tempo. O segundo problema é que a coleta de dados tem de ocorrer em locais apropriados (frigoríficos), além de exigir uma cooperação íntima entre quem faz a coleta e quem utiliza os dados para a avaliação genética. Este procedimento também induz uma fonte de erros, pois uma falha na transferência de um dos dados pode prejudicar o processo por inteiro e esses erros por serem difíceis de ser detectados, e quando detectados, nem sempre podem ser corrigidos (Faria et al., 2007).

Uma alternativa para obtenção dessas medidas seria a utilização do ultrassom por sua rapidez, confiabilidade e alto grau de repetibilidade em suas mensurações na carcaça (Perkins et al., 1997). De acordo com esses mesmos autores, medidas de carcaças com uso da ultrassonografia apresentam considerável acurácia em rendimento de carne e aceitável predição em termos de qualidade. Ainda, segundo Greiner et al. (2003), as mensurações obtidas por ultrassom não são invasivas, apresentam boa acurácia e frequentemente são realizadas quando os animais começam a ganhar peso.

Além disso, medidas de ultrassom podem ser utilizadas em substituição aos dados avaliados diretamente na carcaça, para programas de avaliação genética (Devitt e Wilton, 2001; Kemp et al., 2002). Também, oferecem uma maneira relativamente barata de se obter dados de carcaça de animais vivos, com uma acurácia suficiente para estimar valores genéticos (Reverter et al., 2000).

Ademais, a tecnologia da ultrassonografia é uma ferramenta poderosa que pode ajudar os produtores brasileiros a se manterem competitivos no mercado internacional, oferecendo a qualidade requerida pelos diferentes mercados consumidores, com maior eficiência do sistema produtivo, no melhoramento genético da carcaça e consequentemente no produto final da carne (Maldonado, 2007).

Desta forma, esta revisão tem como objetivo informar sobre o uso do ultrassom na avaliação de carcaça bovina, pontuar os principais indicadores de medidas na carcaça e sua avaliação, além de apresentar as vantagens e limitações do seu uso. 


\section{Desenvolvimento}

O Ultrassom na Avaliação da Carcaça

A utilização do ultrassom para estimar a composição da carcaça in vivo em bovinos não é recente no Brasil, sendo que os primeiros estudos com bovinos de corte foram divulgados na década de 90. Foi utilizado pela primeira vez no Brasil em animais da raça Nelore em 1992, em uma prova de ganho de peso. O objetivo principal da utilização da técnica de ultrassonografia é obter, de forma rápida e barata, informações de carcaça que permitam a avaliação da composição corporal de animais vivos, subsidiando a seleção de animais com carcaças uniformes e específicas para determinados mercados (Tarouco et al., 2005).

Em bovinos a técnica da avaliação de carcaça pelo ultrassom partiu da necessidade de avaliar animais reprodutores. Entretanto, foi somente a partir de 2000 que essa tecnologia começou a ser inserida nos programas de melhoramento genético de bovinos de corte, principalmente da raça Nelore (Lôbo, 2004).

No início do surgimento do ultrassom, essas máquinas utilizavam transdutor único - modo A, sendo a mensuração realizada em apenas um ponto e a aplicação restrita a mensuração da espessura de gordura e da profundidade do músculo. Com o passar dos anos, múltiplos transdutores dispostos linearmente surgiram, aumentando assim a capacidade do ultrassom - modo B. Imagens bidimensionais dos tecidos e órgãos passaram a ser vistas no monitor e novas medidas puderam ser realizadas como espessura de gordura, profundidade e área de olho de lombo. Essa técnica de ultrassom consiste em uma alta freqüência de ondas sonoras, acima da capacidade auditiva humana (16.000 ciclos/segundo). Geralmente, para animais vivos, são utilizadas freqüências entre 1,0 e 5,0 MHz, sendo mais utilizadas frequiências entre 3 e $3,5 \mathrm{MHz}$ para avaliação de carcaça, possibilitando a identificação quantitativa dos tecidos muscular e adiposo através da diferença de impedância acústica (Houghton e Turlington, 1992).

A ultrassonografia vem sendo usada, basicamente, para quatro finalidades dentro das técnicas de avaliação in vivo de carcaças: avaliação da composição corporal dos reprodutores (machos e fêmeas) e de seus descendentes; avaliação dos lotes (grupos contemporâneos) quanto à deposição de gordura, por exemplo, para fins de determinação do momento ótimo de abate; seleção, na fazenda, do gado para mercado e abate, sendo uma ferramenta extra para auxiliar no processo de seleção/descarte no rebanho; valorização do gado comercial no mercado, em particular no mercado de leilões, valorização de lotes e de reprodutores com características positivas para rendimento de carcaça (Cross e Belk, 1994; Maldonato, 2007).

Principais Indicadores de Medidas na Carcaça pelo Ultrassom

Atualmente, existem três principais indicadores de medidas em carcaças de animais vivos que permitem a coleta de informações por ultrassom: espessura de gordura subcutânea (EGS), área de olho de lombo (AOL) e gordura de marmoreio (MAR) (Suguisawa et al., 2003).

A espessura de gordura subcutânea representa a gordura localizada entre a $12^{\mathrm{a}}$ e $13^{\mathrm{a}}$ costela, logo acima do músculo longissimus dorsi. A medida da EGS deve ser obtida a $3 / 4$ da altura do músculo longissimus dorsi. A EGS tem grande importância na industrialização da carne, sendo fundamental no processo de resfriamento da carcaça. Este processo por sua vez deve ser feito de forma lenta e gradual para não causar encurtamento das fibras muscular pelo frio e, por conseguinte, o endurecimento da carne. A falta de gordura de cobertura permite perda excessiva de água, ocasionando, além da perda de peso, o escurecimento da carne durante o período de resfriamento. A consequiência é a diminuição do peso dos cortes comerciais e menor qualidade da carne (Mourão et al., 2007).

A efetividade da uniformização de lotes de animais em confinamento pôde ser observada por vários autores com base na espessura de gordura subcutânea avaliada por ultrassom, com a finalidade de uma maior padronização das características de carcaças e redução dos custos de produção (Delehant et al., 1997; Trenkle e Liams, 1997).

A espessura de gordura subcutânea, quando reduzida em animais para abate, além de influenciar negativamente os atributos de qualidade da carne, também causa perdas aos produtores em razão dos descontos impostos pelos frigoríficos (Silva et al., 2006). Com a maior deposição de gordura subcutânea pode-se agregar maior valor aos animais e proporcionar melhor remuneração ao produtor, uma vez que alguns frigoríficos já pagam $2 \%$ a mais por carcaças de animais Zebu com até 36 meses de idade e espessura de gordura subcutânea de 3-6 mm (Dias, 2011). 
A área de olho de lombo (AOL) é uma medida também realizada sobre o músculo longissimus dorsi, entre a $12^{\mathrm{a}}$ e $13^{\mathrm{a}}$ costela e prediz a quantidade total de carne, sendo utilizada para calcular o rendimento da carcaça (Luchiari Filho, 2000). O autor relatou ainda que a AOL tem sido relacionada à musculosidade e como indicador de rendimento dos cortes de alto valor comercial, além da medida apresentar uma correlação positiva com a porção comestível da carcaça.

O marmoreio ou gordura intramuscular é uma medida, em porcentagem, tomada diretamente sobre o músculo longissimus dorsi entre a $11^{\mathrm{a}}, 12^{\mathrm{a}}$ e $13^{\mathrm{a}}$ costelas e serve para predizer a quantidade de gordura intramuscular ali depositada (Dibiasi et al., 2010).

A área de olho de lombo, a espessura de gordura subcutânea e o marmoreio são características mensuradas por ultrassonografia que estão relacionadas ao ganho de peso diário, rendimento de carcaça, precocidade de acabamento, sabor e suculência da carne (Cartaxo et al., 2011). Ainda, segundo Williams (2002), essas medidas podem ser empregadas na identificação do melhor ponto de abate dos animais, de acordo com as exigências da indústria frigorífica.

A utilização da ultrassonografia com o objetivo de estimar a proporção de músculo e quantidade de gordura é mais acurada do que o peso vivo e outras características facilmente medidas (Wilson, 1992).

Avaliação das Medidas Obtidas In Vivo por Ultrassom e as Obtidas na Carcaça

O conhecimento dos parâmetros genéticos, principalmente a herdabilidade e as correlações com outras características são indispensáveis na determinação do sucesso no programa de seleção em bovinos de corte (Faria et al., 2007).

Em programas de melhoramento genético, a área de olho de lombo, a espessura de gordura subcutânea e o marmoreio são importantes por estarem correlacionadas com o rendimento de cortes cárneos e também por apresentarem valores de herdabilidade estimada de média a alta, sugerindo que possam ser modificadas por seleção (MacNeil et al., 2010).

Greiner et al. (2003) avaliando características da carcaça in vivo de novilhos por ultrassom e pós-abate encontraram coeficientes de correlação entre a área de olho de lombo e a espessura de gordura subcutânea in vivo e as mesmas medidas na carcaça de 0,89 e 0,86, respectivamente. Ou seja, apesar de diversos fatores influenciarem nas medidas de área de olho de lombo e espessura de gordura de cobertura ainda assim são conseguidos bons coeficientes de correlação. Da mesma maneira Prado et al. (2004) concluíram que a avaliação da área de olho de lombo obtida por ultrassom proporcionou alto grau de confiança (80\%) em comparação à avaliação na carcaça de bovinos de corte, mostrando ser um método viável para predição desta característica no animal vivo.

As correlações encontradas para as características da carcaça ainda se mostram um tanto conflitantes na literatura, porém espera-se valores muito superiores hoje em dia, devido ao maior desenvolvimento da tecnologia da ultrassonografia e do maior conhecimento dos fatores que afetam as características das carcaças no abate (Suguisawa et al., 2003).

Vantagens e Limitações no Uso do Ultrassom

São várias as vantagens da técnica de ultrassonografia: pode ser usada nos animais vivos; pode ser utilizada nas plantas de abate antes da remoção do couro; pode predizer com precisão características relacionadas à palatabilidade (ex. marmoreio); não oferece danos a saúde; poderia levar à completa automação da classificação das carcaças e remover o elemento do erro humano (Cross e Belk, 1994).

Além disso, a utilização do ultrassom em tempo real traz como benefícios: uma objetiva predição da carne magra e gordura nos animais vivos; a habilidade de, objetivamente, medir a percentagem de gordura intramuscular no animal vivo; a avaliação da percentagem de gordura intramuscular a partir da área de olho de lombo para determinar o grau de qualidade das carcaças (marmoreio); a informação da composição dos animais vivos, eliminando o custo e tempo necessário pelo teste de progênie tradicional para o mérito de carcaça (Wilson, 1992).

Vários estudos mostram boas correlações entre as medidas realizadas por ultrassom pré-abate e as respectivas medidas na carcaça (Greiner et al., 2003; Suguisawa et al., 2003; Tarouco et al., 2005; Silva et al., 2006; Dibiasi et al., 2010; Son e Lee, 2013). Porém, Perkins et al. (1997) citam que as correlações entre as medidas de ultrassom e na carcaça podem ser influenciadas por limitações tecnológicas (aparelhos), experiência do técnico, nível de gordura e músculo, sexo e idade do animal, 
mudança nas características dos tecidos pós-morte, remoção da gordura junto com o couro e deslocamento dos músculos em relação ao esqueleto.

Waldner et al. (1992) também afirmaram que diferenças devido a instrumentos, operadores, espessura do couro, comprimento do pêlo, peso e nível de acabamento e corte da carcaça contribuem para essa variação de resultados e induzem alguns pesquisadores a concluírem que alguns equipamentos de ultrassom são insuficientemente acurados e inconsistentes para uso em pesquisa ou na indústria. Entretanto, é importante ressaltar que as diferenças atribuídas a equipamentos são mais importantes em se tratando de medidas de gordura intramuscular, com menor influência nas medidas de área de olho de lombo e espessura de gordura subcutânea.

Os mesmos autores, ao avaliarem a utilização do ultrassom em bovinos da raça Brangus em diversos períodos de abate, com as medidas realizadas por vários operadores com diferentes níveis de experiência, afirmaram que a área de olho de lombo (AOL) foi subestimada por todos os operadores, independentemente do nível de experiência.

Alguns autores sugeriram que determinações ultrassônicas da espessura de gordura subcutânea são mais exatas que medidas de AOL (Realini et al., 2001; Williams, 2002) e, entre os fatores que influenciam a precisão destas medidas destacam-se a experiência do técnico com o ultrassom e as alterações post-mortem (Herring et al., 1994; Greiner et al., 2003; Suguisawa et al., 2003; Tarouco et al., 2005).

Assim, para que haja um maior aproveitamento desse equipamento é necessário que alguns aspectos sejam considerados, como: disponibilidade de equipamentos de alta qualidade e de técnicos devidamente treinados e certificados para obtenção de imagens de boa qualidade e para sua correta interpretação (Wilson, 1992).

\section{Considerações Finais}

A ultrassonografia é uma ferramenta que tem sido largamente utilizada para avaliar características de composição de carcaça em animais vivos. Além disso, a aplicação dessa tecnologia tem sido importante na seleção genética, na modelagem do crescimento dos tecidos, na avaliação da condição corporal e também no desenvolvimento de sistemas de pagamento baseados em mérito da carcaça, possibilitando assim a produção de um animal que atenda o mercado consumidor.

\section{Referências}

Cartaxo, F.Q.; Sousa, W.H. de; Cézar, M.F.; Costa, R.G.; Cunha, M. das G.G.; Gonzaga Neto, S. Características de carcaça determinadas por ultrassonografia em tempo real e pós-abate de cordeiros terminados em confinamento com diferentes níveis de energia na dieta. Revista Brasileira de Zootecnia, 40(1): 160-167, 2011.

Cross, H.R.; Belk, K.E. Objective measurement of carcass and meat quality. Meat Science, 36(12): 191-202, 1994.

Delehant, T.M.; Dahlke, G.R.; Hoffman, M.P.; liams, J.C.; Rouse, G.H.; and Wilson, D.E. Iowa State University. Using real-time ultrasound during the feeding period to predict cattle composition. Beef Research Report, 1997. Disponível em: <http://lib.dr.iastate.edu/beefreports_1997/5> . Acesso em: 01 jun. 2017.

Devitt, C.J.B.; Wilton, J.W. Genetic correlation estimates between ultrasound measurements on yearling bulls and carcass measurements on finished steers. Journal of Animal Science, 79(11): 2790-2797, 2001.

Dias, F. Associação Nacional dos Confinadores. Impactos do aumento de peso e acabamento da carcaça sobre os custos de processamento e valor comercial da carne de bovinos. Assocon, 2006. Disponível em: $<$ http://www.assocon.com.br/pdf/pdf_assoco n3.pdf $>$. Acesso em: 01 jun 2017.

Dibiasi, N.F.; Tholon, P.; Barrozo, D.; Fries, L.A.; Queiroz, S.A. Estimativas de correlações genéticas entre características de carcaça medidas por ultrassonografia e por escores visuais em touros Brangus. ARS Veterinária, 26(1): 32-37, 2010.

Faria, C.U.; Magnabosco, C.U.; Borjas, A.R.; Lôbo, R.B.; Bezerra, L.A.F. Inferência bayesiana e sua aplicação na avaliação genética de bovinos da raça nelore: revisão bibliográfica. Ciência Animal Brasileira, 8(1): 75-86, 2007.

Greiner, S.P.; Rouse, G.H.; Wilson, D.E. The relationship between ultrasound measurements and carcass fat thickness and longissimus muscle area in beef cattle. Journal of Animal Science, 81(3): 676-682, 2003. 
Herring, W.O.; Miller, D.C.; Bertrand, J.K.; Benyshek, L.L. Evaluation of machine, technician, and interpreter effects on ultrasonic measures of backfat and longissimus muscle area in beef cattle. Journal of Animal Science, 72(9): 2216-2226, 1994.

Houghton, P.L.; Turlington, L.M. Application of ultrasound for feeding and finishing animals: A review. Journal of Animal Science, 70(3): 930941, 1992.

Kemp, D.J.; Herring, W.O.; Kaiser, C.J. Genetic and environmental parameters for steer ultrasound and carcass traits. Journal of Animal Science, 80(6): 1489-1496, 2002.

Lôbo, R.B. Avaliação genética de touros e matrizes da raça Nelore: Sumário 2004. Ribeirão Preto: GEMAC - Departamento de Genética - FMRPUSP, 2004. 122p.

Luchiari Filho, A. Pecuária da carne bovina. 1ed. São Paulo: LinBife, 2000, 134p.

MacNeil, M.D; Nkrumah, J.D; Woodward, B.W; Northcutt, S.L. Genetic evaluation of Angus cattle for carcass marbling using ultrasound and genomic indicators. Journal of Animal Science, 88(2): 517-522, 2010.

Maldonado, F. Utilização da ultra-sonografia para predição de características de carcaças bovinas. Infobibos, 2007. Disponível em: <http://www.infobibos.com/Artigos/2007_2/ult rasonografia/index.htm>. Acesso em: 01 jun. 2017.

Mourão, G.B.; Ferraz, J.B.S.; Eler, J.P.; Balieiro, J.C.C.; Bueno, R.S.; Mattos, E.C.; Figueiredo, L.G.G. Genetic parameters for growth traits of a Brazilian Beef (Bos taurus x Bos indicus) composite. Genetics and Molecular Research, 6(4): 1190-2000, 2007.

Perkins, T.L., Paschal, J.C., Tipton, N.C., La Zerda, M.J. Ultrasonic Prediction of Quality Grade and Percent Retail Cuts in Beef Cattle. Journal of Animal Science, 75(1): 178-182, 1997.

Prado, C.S.; Pádua, J.T.; Corrêa, M.P.C.; Ferraz, J.B.S.; Miyagi, E.S.; Resende, L.S. Comparação de diferentes métodos de avaliação da área de olho-de-lombo e cobertura de gordura em bovinos de corte. Ciência Animal Brasileira, 5(3): 141-149, 2004.

Realini, C.E.; Willians, R.E.; Pringle, T.D. Gluteus medius and rump fat depths as additional ultrasound measurements for predicting retail product and trimmable fat in beef cattle. Journal of Animal Science, 79(6): 1385-1387, 2001.

Reverter, A.; Johnston, D.J.; Graser, H.U.; Wolcott, M.L.; Upton, W.H. Genetic analyses of liveanimal ultrasound and abattoir carcass traits in Australian Angus and Hereford cattle. Journal of Animal Science, 78(7): 1786-1985, 2000.

Silva, S.L.; Leme, P.R. ; Putrino, S.M. ; Lanna, D. P. D. Alterações nas características de carcaça de tourinhos Nelore, avaliadas por ultra-som. Revista Brasileira de Zootecnia, 35(2): 607612, 2006.

Son, J.; Lee, D. Estimation of Genetic Correlations and Selection Responses for Carcass Traits between Ultrasound and Real Carcass Measurements in Hanwoo Cows. Journal of Animal Science and Technology, 55(6): 501508, 2013.

Suguisawa, L.; Mattos, W.R.S.; Oliveira, H.N. de; Silveira, A.C.; Arrigoni, M. de B.; Haddad, C.M.; Chardulo, L.A.L.; Martins, C. L. Ultrasonography as a Predicting Tool for Carcass Traits of Young Bulls. Scientia Agricola, 60(4): 779-784, 2003.

Tarouco, J.U.; Lobato, J.F.P.; Tarouco, A.K.; Massia, G.S. Relação entre medidas ultra-sônicas e espessura de gordura subcutânea ou área de olho-de-lombo na carcaça em bovinos de corte. Revista Brasileira de Zootecnia, 34(6): 20742084, 2005.

Trenkle, A.; Liams, J.C. Iowa State University. Potential value of ultrasound to sort feeder cattle into more uniform groups for finishing and marketing. Beef Research Report, 1997. Disponível em: <http://lib.dr.iastate.edu/beefreports_1997/4/>. Acesso em: 01 jun 2017.

Waldner, D.N.; Dikeman, M.E.; Schalles, R.R.; Olson, W.G.; Houghton, P.L.; Unruh, J.A.; Corah, L.R. Validation of real-time ultrasound technology for predicting fat thicknesses, longissimus muscle areas, and composition of Brangus bulls from 4 months to 2 years of age. Journal of Animal Science, 70(10): 3044-3054, 1992.

Williams, A.R. Ultrasound applications in beef cattle carcass research and management. Journal of Animal Science, 80(2): 183-188, 2002.

Wilson, D.E. Application of ultrasound for genetic improvement. Journal of Animal Science, 70(3): 973-983, 1992. 